little work on exploring ways of capitalizing on key image characteristics. In particular, volumetric displays provide considerable freedom in viewing position, and support both vertical and horizontal motion parallax, which means that observers can move and change their view of an image in a wholly natural way.

Consequently, these devices offer exciting, and largely unexplored, opportunities to advance spatial imaging (in areas such as neurosurgery) and dynamic imaging (in fields including fluid dynamics, robotics and sports training). With regard to the latter, there is a need to better support the visualization of complex forms of 3D motion ${ }^{8}$. Moreover, creating volumetric images in the air enables direct interaction, thereby allowing, for example, 3D design tasks to be carried out in a natural way in $3 \mathrm{D}$ space.

Smalley and colleagues' approach could provide the foundation for the next generation of volumetric displays. Such devices will not only enhance our understanding of complex spatial and geometric dynamics, but also support innovative user interaction.

Barry G. Blundell is with the University of Derby Online Learning (UDOL), University of
Derby, Derby DE22 1GB, UK.

e-mail: barry.blundell@physics.org

1. Smalley, D. E. et al. Nature 553, 486-490 (2018)

2. Luzy, E. \& Dupuis, C. Procédé pour obtenir des projections en relief. French Patent 461,600 (1914).

3. Blundell, B. G. Enhanced Visualization: Making Space for 3-D Images 68 (Wiley, 2007).

4. Blundell, B. G. \& Schwarz, A. Volumetric ThreeDimensional Display Systems 72-91 (Wiley, 2000).

5. Davis, E. J. Aerosol Sci. Technol. 26, 212-254 (1997)

6. Adamson, A. W. Method and apparatus for generating three-dimensional patterns. US Patent 3,609,706 (1971).

7. Adelman, A. H. \& Lewis, J. D. Method and apparatus for generating three-dimensional patterns. US Patent 3,609,707 (1971)

8. Blundell, B. G. 3D Res. 8, 11 (2017).

\section{Ageing-related receptors resolved}

\begin{abstract}
Ageing is a regulated process in which hormones have pivotal roles. Crystal structures of two hormone co-receptors should be informative for drug discovery focused on age-related disorders. SEE ARTICLE P.461 \& LETTER P.501
\end{abstract}

\section{MAKOTO KURO-O}

$\mathrm{I}$ n Greek mythology, three goddesses known as the Fates govern the lifespan of each person. Klotho, Lachesis and Atropos are the spinner, the allotter and the cutter of the thread of life, respectively. So when a genetic mutation was identified in mice that undergo premature ageing ${ }^{1}$, the gene involved was fittingly named klotho. The protein it encodes, $\alpha$-klotho, and a sister protein called $\beta$-klotho, are high-affinity co-receptors for certain members of the fibroblast growth factor (FGF) family of signalling proteins ${ }^{2}$, but their means of action has not been well characterized. Two papers ${ }^{3,4}$ in this issue describe crystal structures of FGF-klotho complexes, not only providing a basis for understanding how klothos act, but also opening up avenues for structure-based drug design.

$a$-Klotho is a membrane-spanning protein expressed predominantly in the kidney, as well as in the brain. Mice lacking $\alpha$-klotho exhibit a range of signs associated with ageing, including hearing loss, impaired cognition and organ atrophy ${ }^{5}$. They also have elevated blood phosphate levels. However, the protein's function on the molecular level was unclear, until mice lacking FGF23 were characterized ${ }^{6}$.

FGF23 is one of the three endocrine FGFs, which act as hormones, secreted by one organ to regulate the function of another. Specifically, FGF23 is secreted from bones after phosphate intake and acts in the kidney to inhibit phosphate reabsorption in urine, thereby maintaining the body's phosphate balance. Mice lacking FGF23 have elevated phosphate levels owing to impaired phosphate excretion, and exhibit features associated with ageing ${ }^{6}$. This striking similarity to mice lacking $\alpha$-klotho led researchers to discover ${ }^{2}$ that $\alpha$-klotho forms a complex with the membrane-spanning protein FGF receptor 1c (FGFR1c), acting as a co-receptor to recruit FGF23 and so triggering FGF signalling

In the first of the current studies, Chen et al. $^{3}$ (page 461) solved the crystal structure of FGF23 in complex with the ligand-binding domain of FGFR1c and the extracellular domain of $\alpha$-klotho. The structure revealed that $\alpha$-klotho (aptly, given its namesake) sends out a long receptor-binding arm (RBA) that acts as a thread to capture the ligand-binding domain of FGFR1c. Indeed, when the authors generated $\alpha$-klotho lacking the RBA, the mutant protein failed to capture FGFR1c or to help FGF23 to activate FGF signalling.

Chen and colleagues showed that FGF23 fits into the groove created between $\alpha$-klotho and FGFR1c. The globular amino-terminal region and the rod-like carboxy-terminal region of FGF23 face FGFR1c and $\alpha$-klotho, respectively (Fig. 1). By promoting formation of this complex, $\alpha$-klotho enables strong interactions between FGF23 and FGFR1c, which otherwise interact only weakly.

Like $\alpha$-klotho, $\beta$-klotho functions as a co-receptor for endocrine FGFs, forming a complex with FGFR1c to bind FGF21, and with FGFR4 to bind FGF19 (refs 7,8). FGF19 is secreted from the intestine after feeding, and acts in the liver to suppress bile-acid synthesis. FGF21 is secreted from the liver following fasting, and acts in fat cells and the brain to induce metabolic adaptation to fasting and responses to stress ${ }^{5}$. Although FGFRs are expressed in a wide range of tissues, the tissuespecific expression of $\beta$-klotho in the liver, fat and brain restricts the target organs of these endocrine FGFs.

In the second study, Lee et al. ${ }^{4}$ (page 501)

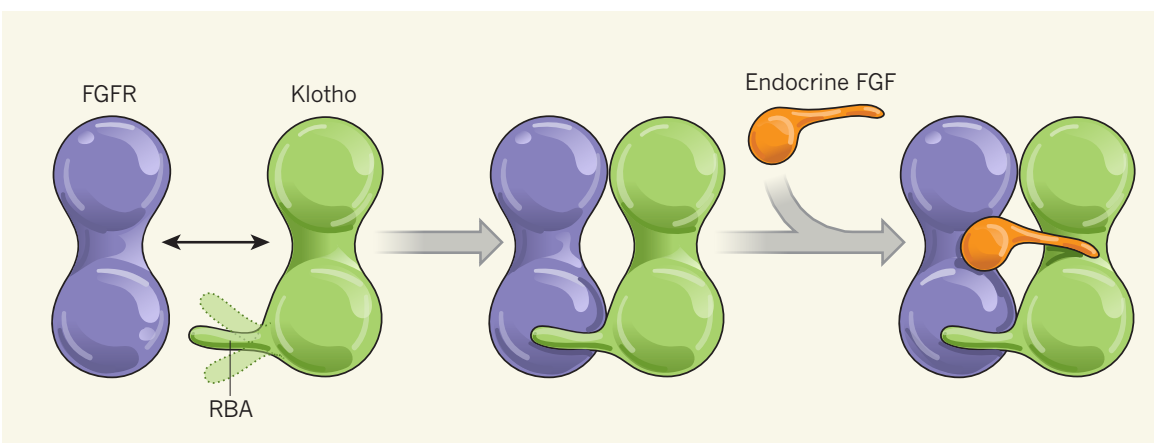

Figure 1 | Structures reveal the mode of action for klotho proteins. Two groups $\mathrm{s}^{3,4}$ have produced crystal structures of the extracellular domains of klotho proteins, either alone, in complex with 'endocrine' fibroblast growth factors (FGFs), or in complex with both endocrine FGFs and the ligandbinding domains of FGF receptors (FGFRs). As this simplified schematic shows, the klotho proteins seem to have an intrinsically disordered receptor-binding arm (RBA) with which they capture FGFRs (interaction indicated by double-headed arrow). The RBA enables formation of a stable complex, with FGFs fitting into the groove between the other two proteins. 
resolved the crystal structure of $\beta$-klotho's extracellular domain when bound to and when free from FGF21, in the absence of FGFRs. Like FGF23, the C-terminal region of FGF21 fits into the groove in $\beta$-klotho. However, the authors could not solve the structure of some regions in $\beta$-klotho, including that corresponding to the RBA in $\alpha$-klotho. This suggests that the RBAs of klotho proteins are intrinsically disordered and unable to fold stably unless bound to FGFRs. The fact that intrinsically disordered proteins can interact with multiple proteins ${ }^{9}$ implies that the RBAs of klotho proteins could capture other partners besides FGFRs. This might explain why the extracellular domain of a-klotho, which can be released into the extracellular space, has been reported to have FGF-independent activity, regulating several ion channels and transporters, along with other growth factors and their receptors ${ }^{5}$.

Another proposed FGF-independent activity for the klothos is as carbohydrate-binding proteins called lectins. Klothos belong to a family of enzymes that cut sugar chains ${ }^{1}$, but not all of the amino-acid residues essential for this enzymatic activity are found in the klothos. Thus, klothos might bind to, but not cut, specific carbohydrates. Lee and colleagues' structure of $\beta$-klotho leaves open the possibility that this protein interacts with particular sugar chains. By contrast, Chen and co-workers' structure of FGF23-a-klotho-FGFR does not fit with the idea of $\alpha$-klotho acting as either an enzyme or a lectin. However, it might be that in the absence of FGFRs, the structure of a-klotho would provide a different point of view. Alternatively, it is possible that the two klothos have different FGF-independent activities.

FGF-klotho signalling has key roles in ageing and age-related disorders. The new structures could be used to develop drugs to treat disorders of ageing, using structurebased drug design to identify targets in FGF-klotho-FGFR complexes. For instance, consider chronic kidney disease $(\mathrm{CKD})^{5,10}-\mathrm{a}$ common state of impaired renal function that often occurs as a complication of high blood pressure or diabetes. People with CKD exhibit many of the same symptoms as mice lacking $a$-klotho, including disturbed phosphate metabolism and increased risk of death ${ }^{5,10}$. Placing mice lacking FGF23 or $\alpha$-klotho on a low-phosphate diet reduces the phosphate retention and premature ageing normally seen in these animals, indicating that phosphate increases accelerate ageing ${ }^{10}$. Thus, drugs that target FGF-klotho-FGFR complexes to improve phosphate metabolism might be useful to treat CKD.

A second example lies in the targeting of FGF21 complexes. FGF21 overexpression extends lifespan in mice $^{11}$, and this protein has been dubbed an 'anti-ageing' hormone. Lee et al. demonstrated that they could increase the potency of FGF21 by introducing genetic mutations designed to increase the protein's affinity for $\beta$-klotho. Further analyses such as this could provide a way to explore anti-ageing medicines more generally.

Makoto Kuro-o is in the Division of Anti-Ageing Medicine, Center for Molecular Medicine, Jichi Medical University, Shimotsuke, Tochigi 329-0498, Japan. e-mail:mkuroo@jichi.ac.jp

1. Kuro-o, M. et al. Nature 390, 45-51 (1997)

2. Kurosu, H. et al. J. Biol. Chem. 281, 6120-6123 (2006).
3. Chen, G. et al. Nature 553, 461-466 (2018).

4. Lee, S. et al. Nature 553, 501-505 (2018).

5. Hu, M. C., Shiizake, K., Kuro-o, M. \& Moe, O. W. Annu. Rev. Physiol. 75, 503-533 (2013).

6. Shimada, T. et al. J. Clin. Invest. 113, 561-568 (2004)

7. Ogawa, Y. et al. Proc. Natl Acad. Sci. USA 104, 7432-7437 (2007).

8. Kurosu, H. et al. J. Biol. Chem. 282, 26687-26695 (2007).

9. Wright, P. E. \& Dyson, H. J. J. Mol. Biol. 293, 321-331 (1999).

10.Kuro-o, M. Nature Rev. Nephrol. 9, 650-660 (2013)

11.Zhang, Y. et al. eLife 1, e00065 (2012).

This article was published online on 17 January 2018.

\section{A beacon at the dawn of the Universe}

\section{Quasars are the brightest continuously emitting sources of radiation in the Universe. Measurements of the most distant quasar ever detected reveal details about the evolution and structure of the early Universe. SEE LETTER P.473}

\section{EILAT GLIKMAN}

$\mathrm{S}$ ince their discovery ${ }^{1}$ in 1963 , astronomical objects called quasars have been among our most powerful probes of the early Universe. Initially seen as mysterious sources of extreme luminosity, quasars are now known to be supermassive black holes that are voraciously consuming gas from their immediate surroundings, emitting large amounts of radiation in the process. On page 473, Bañados et al. ${ }^{2}$ report observations of the most distant quasar found so far. The light detected from this object was emitted when the Universe was a mere 690 million years old — just $5 \%$ of its current age.

Almost 90 years ago, the astronomer Edwin Hubble discovered that the Universe is expanding ${ }^{3}$. The expansion stretches light waves travelling through space, such that light that was emitted from a distant source as blue might be detected as red. This phenomenon is called redshift, and is associated with both distance and time: the larger the redshift, the farther away the source was when it emitted its light, meaning that the light was emitted at an earlier time.

If we rewind the expansion, we find that the

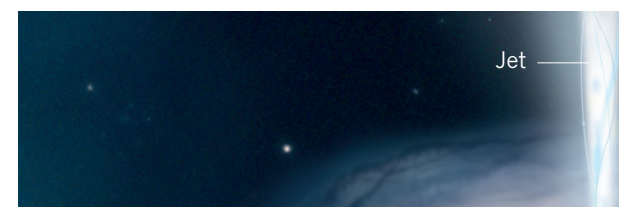

Accretion disk .

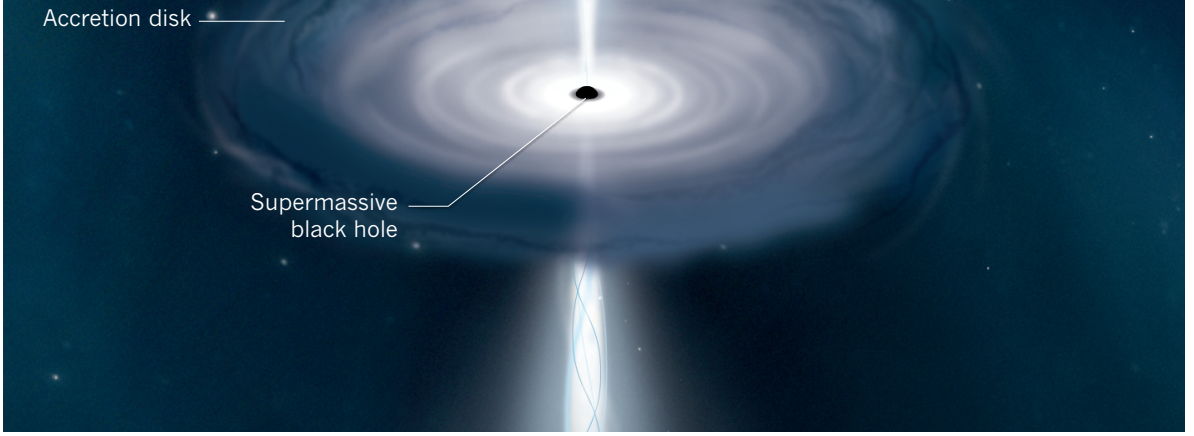

Figure 1 | Emission from a quasar. Quasars are extremely luminous astronomical objects that comprise a supermassive black hole surrounded by an orbiting disk of gas called an accretion disk. As material in the disk is pulled towards the black hole, energy is released in the form of electromagnetic radiation and, in some cases, as beams of charged particles called jets. Bañados et al. ${ }^{2}$ report observations of the most distant quasar identified so far, the light of which was emitted when the Universe was only $5 \%$ of its current age. 